\title{
EQUILIBRIO DE ADSORCIÓN DEL COLORANTE AZUL DE METILENO SOBRE CARBÓN ACTIVADO
}

\section{ADSORPTION EQUILIBRIUM OF METHYLENE BLUE DYE ON ACTIVATED CARBON}

\author{
Grey Castellar ${ }^{1}$, Edgardo Angulo ${ }^{2}$, Alejandra Zambrano ${ }^{3}$, Dianis Charris ${ }^{4}$
}

${ }^{1}$ M.Sc. Universidad Autónoma del Caribe, Grupo de Investigación en Química Ambiental, Barranquilla, Atlántico. Email: grey.castellar@uac.edu.co; ${ }^{2}$ M.Sc. Universidad del Atlántico, Grupo de Investigación en Biotecnología de Microalgas. Email: edgangulo20@gmail.com; ${ }^{3,4}$ Esp. Universidad del Atlántico, Grupo de Investigación en Biotecnología de Microalgas.

Rev. U.D.C.A Act. \& Div. Cient. 16(1): 263 - 271, 2013

\section{RESUMEN}

Los colorantes sintéticos son una clase importante de compuestos orgánicos que, con frecuencia, se encuentran en el medio ambiente, como resultado de su amplio uso industrial, convirtiéndose en agentes que, desafortunadamente, afectan la ecología. La disposición de estos colorantes a los recursos de agua se debe evitar; para tal efecto, se utilizan varias técnicas de tratamiento, siendo la adsorción una de las más empleadas. Este artículo presenta un estudio sobre la adsorción del colorante azul de metileno (AM) en disolución acuosa sobre carbón activado granular (CAG) a $25^{\circ} \mathrm{C}$. Se realizaron experimentos por lote para determinar el efecto de la concentración inicial (300-1100 $\mathrm{mg} \mathrm{dm}^{-3}$ ) y del $\mathrm{pH}$ (4-8), sobre la capacidad de adsorción y el porcentaje de remoción. Las isotermas de equilibrio fueron analizadas a partir de las ecuaciones de Langmuir y Freundlich usando el coeficiente de correlación. Los datos experimentales muestran un ajuste satisfactorio con el modelo de isoterma de Langmuir. Los valores de capacidad máxima de adsorción de $\mathrm{AM}$ en monocapa obtenidos fueron de 76,3; 79,4; 80,6; 83,3 y $87 \mathrm{mg} \mathrm{g}^{-1}$ a los $\mathrm{pH}$ de 4, 5, 6, 7 y 8, respectivamente.

Palabras clave: Colorantes sintéticos, contaminación, carbón activado, isotermas de adsorción.

\section{SUMMARY}

Synthetic dyes are an important class of organic compounds, which unfortunately are agents affecting the ecology, often found in the environment as a result of their widespread industrial use. The disposal of these dyes into water resources should be avoided, therefore different treatment techniques are used, being adsorption one of the most employed. This paper presents a study on the adsorption of methylene blue dye (MB) in aqueous solution on granular activated carbon
(GAC) at $25^{\circ} \mathrm{C}$. Batch experiments were conducted to determine the effect of the initial concentration $(300-1100 \mathrm{mg}$ $\mathrm{dm}^{-3}$ ) and $\mathrm{pH}(4-8)$, on the adsorption capacity and rate of removal. The results show that the amount of $M B$ removed increases when increasing both the initial concentration and $\mathrm{pH}$. The equilibrium isotherms were analyzed based on the Langmuir and Freundlich equations using the correlation coefficient. The experimental results showed a satisfactory fit to the Langmuir isotherm. The values of maximum adsorption capacity of $M B$ in monolayer obtained were $76.3,79.4,80.6$, 83.3 and $87 \mathrm{mg} \mathrm{g}-1$ at $\mathrm{pH} 4,5,6,7$ and 8 respectively.

Key words: Synthetic dyes, contamination, activated carbon, adsorption isotherms.

\section{INTRODUCCIÓN}

Los colorantes son usados ampliamente en la industria de textiles, de caucho, de papel, de plásticos y de cosméticos, con el propósito de colorear (Malik, 2003). La descarga de aguas coloreadas a los cuerpos de agua causa serios problemas al medio ambiente, como la disminución de la actividad fotosintética, debido a la interferencia en la penetración de la luz (Choy et al. 2004) e inhiben la reacción de los agentes oxidantes (Ramakrishna \& VIraraghavan, 1997). Es importante señalar, además, que algunos colorantes tienen efecto tóxico, cancerígeno (Wang et al. 2005) y mutagénico, por la tendencia a formar quelatos de iones metálicos que producen microtoxocidad, tanto para la vida acuática como humana (Suhas et al. 2007); por lo tanto, antes de verterlos a los cuerpos de agua se hace necesario removerlos (DíazVelázquez et al. 2007; Wachowski et al. 2007).

Se conocen alrededor de 100.000 colorantes comerciales y más de $700.000 t$ métricas se producen anualmente, de las cuales, 35.000-70.000 se descargan en las aguas residuales 
(Tunc et al. 2009; Dafale et al. 2008). Colombia no es ajena a esta realidad y, más aun, con la apertura de nuevos mercados de comercio en el exterior, como los Tratados de Libre Comercio, que se han firmado con varios países, que prevé a futuro un crecimiento industrial, principalmente del sector textil, por lo que se hace indispensable disminuir los impactos negativos al medio ambiente (Peña \& Tobón, 2006).

Las técnicas para la eliminación de colorantes en aguas residuales se pueden dividir en tres importantes categorías: los métodos químicos, biológicos y físicos. El tratamiento químico utiliza agentes coagulantes o floculantes para eliminar el color, pero no es bueno para colorantes que son muy solubles (Shi et al. 2007). Por otro lado, el tratamiento biológico tiene dificultades para eliminar colorantes de efluentes de manera continua; además, se requiere de tiempos más prolongados en los procesos de decoloración-fermentación (Robinson et al. 2001) y, por último, los tratamientos físicos, como la filtración por membrana, la destrucción electroquímica, la irradiación, la ozonización y la adsorción, entre otros, se usan ampliamente para el tratamiento de colorantes en aguas residuales. La mayoría de estos procesos son costosos y conducen a la generación de lodos o la formación de subproductos (Hameed \& El-Khaiary, 2008); sin embargo, se conoce que la adsorción es el método más eficaz, sobre todo si el adsorbente es barato y muestra una alta capacidad de adsorción para la eliminación de colorantes de las aguas residuales (Ahmad \& Alrozi, 2010). Los carbones activados, por su gran porosidad, son usados ampliamente como adsorbentes en las operaciones industriales de purificación y de recuperación química, que se debe a su extensa área superficial, entre 500 y $2000 \mathrm{~m}^{2} \mathrm{~g}^{-1}$, su gran volumen de poro y la presencia de grupos funcionales superficiales, en especial, grupos oxigenados (Bansal et al. 1988).

Esta investigación evaluó la capacidad de adsorción de un carbón activado comercial en la remoción del colorante ácido azul de metileno (AM) en disolución acuosa, modificando el pH y la concentración inicial de las disoluciones estudiadas.

\section{MATERIALES Y MÉTODOS}

Caracterización del carbón activado: La caracterización física del carbón activado, se evaluó, parcialmente, mediante el análisis de textura, a partir de la isoterma de adsorcióndesorción de nitrógeno, a 77 K. Para tal efecto, se utilizó un sortometro Micromeritics Gemini III 2375; la cantidad de carbón activado utilizado fue de 0,1207 g y su desgasificación tuvo lugar a $250^{\circ} \mathrm{C}$. Para las isotermas, se tomaron ochenta (80) puntos de adsorción y veinte (20) de desorción; se empleó el modelo de BET para hallar el área superficial (Ahmad et al. 2007; Shi et al. 2010). En la caracterización química del carbón activado, se determinó el contenido de humedad, de material volátil y de cenizas; de igual manera, se analizó el contenido de carbono, de hidrógeno, de azufre y de oxígeno por diferencia, según las normas ASTM.

Estudio por lote: Para el estudio por lote el carbón activado, se lavó con agua destilada y, posteriormente, se secó a $100^{\circ} \mathrm{C}$, durante 12 horas. Se preparó una disolución "stock" de $3000 \mathrm{mg} \mathrm{dm}^{-3}$ de $\mathrm{AM}$ (fórmula molecular $\mathrm{C}_{16} \mathrm{H}_{18} \mathrm{~N}_{3} \mathrm{ClS}$, masa molar $319,85 \mathrm{~g} \mathrm{~mol}^{-1}$ ), disolviendo la cantidad requerida de AM en agua destilada. A partir de la disolución "stock", se prepararon diluciones de concentraciones entre los $300 \mathrm{mg} \mathrm{dm}^{-3}$ hasta los $1100 \mathrm{mg} \mathrm{dm}^{-3}$, ajustándole el pH a valores comprendidos entre 4,0 - 8,0 \pm 0 , con $\mathrm{HCl}$ y $\mathrm{NaOH}$ 0,1 M. A $1000 \mathrm{mg}$ del carbón activado se le adicionaron $100 \mathrm{~cm}^{3}$ de cada una de las diluciones; luego, se colocaron en un agitador horizontal, a 170rpm, por 16 horas, tiempo estimado para alcanzar la condición de equilibrio (Ahmad et al. 2007), a la temperatura del laboratorio $25^{\circ} \mathrm{C} \pm 1^{\circ} \mathrm{C}$. Finalizada la agitación, se separó el carbón activado por filtración y se analizó la concentración de $\mathrm{AM}$, usando un espectrofotómetro BECKMAN DU 64 UV/VIS a 660nm.

\section{RESULTADOS Y DISCUSIÓN}

Caracterización del carbón activado: A partir de los datos de la isoterma de adsorción-desorción de nitrógeno a $77 \mathrm{~K}$ fue posible calcular las propiedades de textura para el carbón activado utilizado. Para hallar el área superficial, se aplicó el método propuesto por S. Brunauer, P. Emmett y E. Teller, a través de la ecuación de BET (Bruanuer et al. 1938):

$$
\frac{p_{r}}{n^{s}\left(1-p_{r}\right)}=\frac{1}{n_{m} c}+\frac{c-1}{n_{m}} p_{r}
$$

donde es la razón entre la presión de equilibrio $(P)$ y la presión de saturación del nitrógeno $\left(P^{\circ}\right), n^{s}$ es la cantidad adsorbida por unidad de masa $\left(\mathrm{mol} \mathrm{g}^{-1}\right), n_{m}$ es la cantidad necesaria para formar la monocapa y es una constante.

Al graficar $\frac{P_{r}}{n^{s}\left(1-P_{r}\right)} \quad$ versus $P_{r}$ se obtiene el gráfico de BET, cuya porción lineal típicamente se extiende hasta un valor de 0,3 para $P_{r}$.

El valor de $n_{m}$ se calculó a partir de los parámetros intercepto y pendiente de la recta que se ajustó a los datos experimentales. El área superficial específica $\left(\alpha_{\mathrm{BET}}\right)$, se obtuvo a partir de la ecuación 2:

$$
a_{B E T}=L n_{m} a_{m}
$$

donde $L$ es el valor numérico del número de Avogadro y $\alpha_{m}$ es el área transversal ocupada por una molécula de $N_{2}(0,16$ $\mathrm{nm}^{2}$ ). Las propiedades fisicoquímicas, como área superficial específica, material volátil, cenizas, contenido de carbono y otras propiedades, se muestran en la tabla 1. 
Aunque el área superficial del carbón activado empleado en este estudio se puede considerar aceptable, la capacidad de adsorción de los materiales no solamente se puede interpretar en términos de sus propiedades físicas, sino que también influye su química de superficie (Orfão et al. 2006), que juega un papel importante en su rendimiento como adsorbente (Bansal et al. 1988). La química de superficie depende de la presencia de heteroátomos, principalmente oxígeno (Tabla 1), que forma grupos funcionales orgánicos, como ácidos carboxílicos, lactonas, fenoles, carbonilos, quinonas, aldehídos, éteres, anhídridos e, incluso otros (Boehm, 1994), que pueden ser tanto ácidos como básicos y, dependiendo del $\mathrm{pH}$ del medio, afectan la extensión de la adsorción. El alto contenido de cenizas y de humedad presentes en el CAG empleado, disminuyen la cantidad realmente efectiva de adsorbente.

Tabla 1. Principales características del adsorbente.

\begin{tabular}{|c|c|}
\hline Parámetro & Valor \\
\hline $\begin{array}{l}\text { Área superficial }\left(\mathrm{m}^{2} \mathrm{~g}^{-1}\right) \\
\text { (basado en BET) }\end{array}$ & 631 \\
\hline Contenido de cenizas (base seca) (\%) & 13,70 \\
\hline Contenido de humedad (\%) & 15,24 \\
\hline Materia volátil (\%) & 8,41 \\
\hline \multicolumn{2}{|l|}{ Análisis elemental (\%) } \\
\hline C & 78,89 \\
\hline $\mathrm{H}$ & 2,5 \\
\hline$N$ & 0,59 \\
\hline O (por diferencia) & 3,25 \\
\hline S & 1,07 \\
\hline
\end{tabular}

Efecto de la concentración inicial de AM: El efecto de la concentración inicial del colorante en el rango de 300 a 1100mg $\mathrm{dm}^{-3}$ con una dosis de adsorbente de $10 \mathrm{~g} \mathrm{dm}^{-3}$, sobre el porcentaje de remoción y la capacidad de adsorción del CAG, se muestra en la figura 1. Al aumentar la concentración inicial desde $300 \mathrm{mg} \mathrm{dm}^{-3}$ hasta $1100 \mathrm{mg} \mathrm{dm}^{-3}$ a pH 8 , el porcentaje de remoción del AM disminuye desde $99,9 \%$ a $80,2 \%$, respectivamente. Esta misma tendencia, se observa para los otros $\mathrm{pH}$ estudiados. El anterior comportamiento, se debe a la mayor disponibilidad de sitios activos de adsorción a bajas concentraciones, en consecuencia, gran parte de las moléculas de AM pueden ser removidas de la disolución. A medida que la concentración inicial aumenta más moléculas de AM compiten por los sitios disponibles y aun después de haber alcanzado el equilibrio, quedan moléculas en la disolución, lo que reduce el porcentaje de remoción (Ahmad \& Alrozi, 2010; Baskaralingam et al. 2007). En lo que respecta a la capacidad de adsorción, su valor se incrementa desde $30,5 \mathrm{mg} \mathrm{g}^{-1}$ a $87,0 \mathrm{mg} \mathrm{g}^{-1}$, cuando aumenta la concentración inicial desde $300 \mathrm{mg} \mathrm{dm}^{-3}$ hasta $1100 \mathrm{mg} \mathrm{dm}^{-3}$ a pH 8; igual tendencia se muestra para los otros $\mathrm{pH}$. La fuerza motriz de la adsorción es la diferencia de concentración entre el soluto, en el adsorbente y en el soluto, en la disolución. Un gradiente de concentración bajo causa un transporte lento, debido a una disminución del coeficiente de difusión o coeficiente de transferencia de masa; éste mejora al aumentar la concentración inicial, ocasiona un transporte más rápido y, en consecuencia, aumenta la capacidad de adsorción (Ahmad \& Hameed, 2010). Por otro lado, a bajas concentraciones existe menor cantidad de colorante en disolución que a altas concentraciones. Si se emplea la misma masa de carbón, se establece una relación cada vez mayor en la medida que aumenta la concentración inicial (Castellar \& García, 2011; Chatterjee et al. 2005; Chiou \& Li, 2003).

Isotermas de adsorción: Cuando se establece una afinidad entre el carbón activado y el AM, este último es atraído hacia el sólido, mediante diferentes mecanismos, hasta que se alcance un equilibrio entre ambos. Este equilibrio se describe mediante modelos matemáticos, mejor conocidos como isotermas de adsorción, que relaciona la cantidad de AM removido y la que permanece en disolución cuando se alcanza el equilibrio, a una temperatura constante (Ho et al. 2002).

Varios modelos de equilibrio se han desarrollado para ajustar los datos experimentales a las isotermas de adsorción. Los modelos de Freundlich y de Langmuir son los más ampliamente utilizados y se han aplicado en el presente trabajo.

Isoterma de Freundlich: En 1906, Freundlich estudió la adsorción de un soluto sobre carbón activado y encontró una relación de tipo exponencial entre la cantidad de soluto adsorbido y la concentración en equilibrio, que se expresa, mediante la siguiente ecuación (Freundlich, 1906):

$q=k C_{e}^{\frac{1}{n}}$

donde $q$ es la cantidad de soluto removido por unidad de masa de adsorbente $\left(\mathrm{mg} \mathrm{g}^{-1}\right), C_{e}$ es la concentración de AM en el equilibrio $\left(\mathrm{mg} \mathrm{dm}^{-3}\right)$, es la constante de equilibrio ( $\mathrm{mg}$ $\left.\mathrm{g}^{-1}\left(\mathrm{dm}^{3} \mathrm{mg}^{-1}\right)^{-1 / n}\right)$ y $n$ es una constante relacionada con la afinidad entre el adsorbente y el soluto.

La ecuación 3, se puede linealizar aplicando logaritmo natural:

$\ln q=\ln k+\frac{1}{n} \ln C_{e}$

De forma que, representando gráficamente $\operatorname{Inq}$ versus $\operatorname{In} C_{e}$ de los valores de la pendiente y la ordenada al origen, se obtienen los parámetros de la isoterma de Freundlich. 


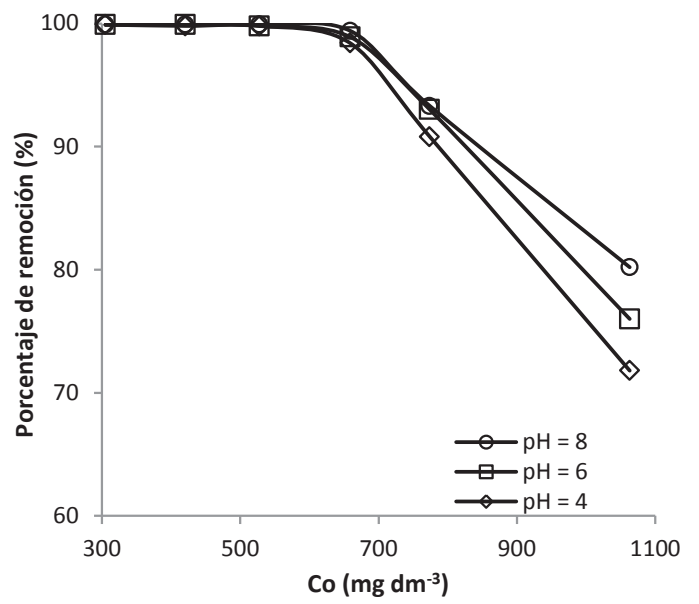

(a)

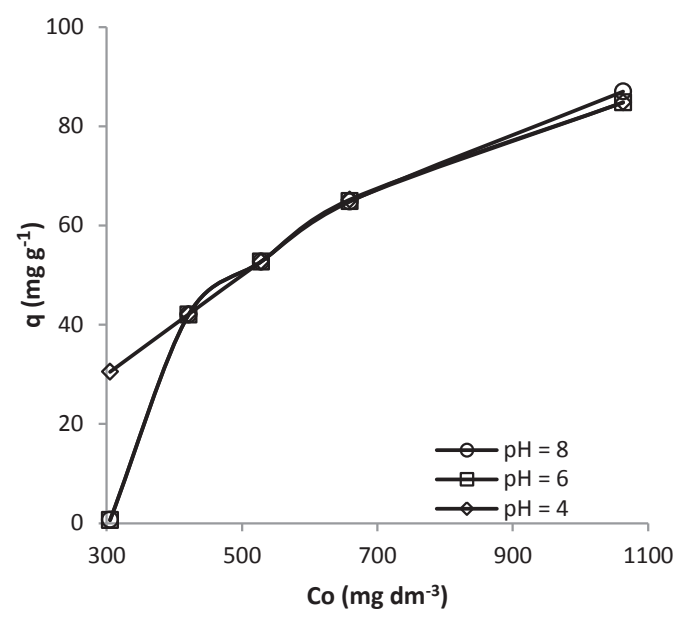

(b)

Figura 1. Efecto de la concentración inicial sobre (a) el porcentaje de remoción y (b) la capacidad de remoción.
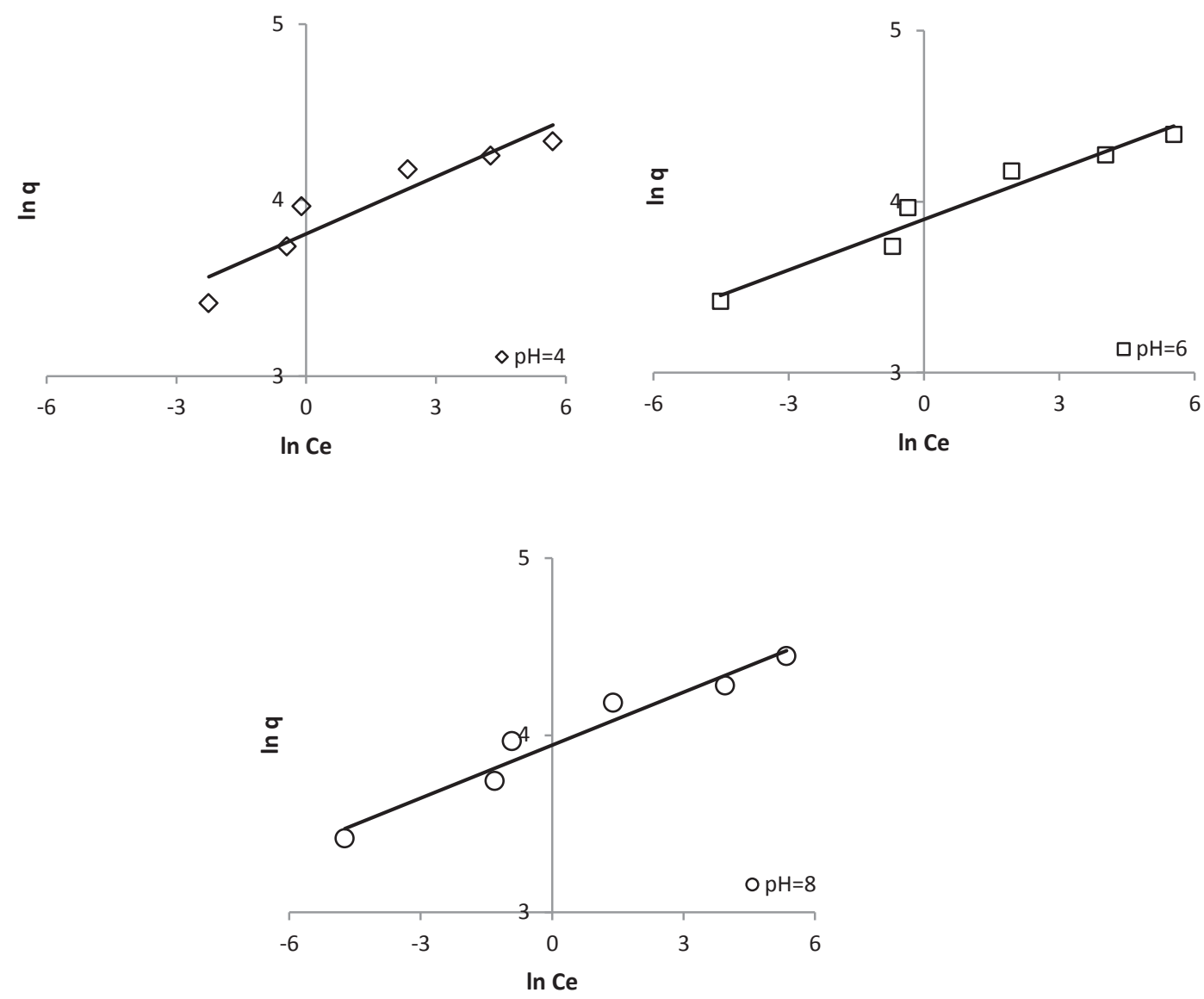

Figura 2. Comparación entre la ecuación linealizada del modelo de Freundlich y los datos experimentales a diferentes pH. 
Tabla 2. Constantes de Langmuir y Freundlich.

\begin{tabular}{|c|c|c|c|c|c|c|}
\hline $\mathbf{p H}$ & $\begin{array}{c}\text { Constantes de Langmuir } \\
\text { Coeficiente de correlación } \\
\left(R^{2}\right)\end{array}$ & $\begin{array}{c}\mathbf{q}_{\text {max }} \\
\left(\mathbf{m g ~ g}^{-1}\right)\end{array}$ & $\begin{array}{c}b \\
\mathbf{d m}^{\mathbf{2}} \\
\left.\mathbf{m g}^{-1}\right)\end{array}$ & $\begin{array}{c}\text { Constantes de Freundlich } \\
\text { Coeficiente de correlación } \\
\left(R^{2}\right)\end{array}$ & $\begin{array}{c}K \\
\left(\mathbf{m g g}^{-1}\left(\mathbf{d m}^{3} \mathbf{m g}^{-1}\right)^{-1 / n}\right)\end{array}$ & $n$ \\
\hline 4,0 & 0,99 & 76,3 & 0,64 & 0,87 & $4, .80$ & 10 \\
\hline 5,0 & 0,99 & 79,4 & 0,56 & 0,93 & 4,10 & 10 \\
\hline 6,0 & 0,99 & 80,6 & 0,59 & 0,95 & 49,2 & 11 \\
\hline 7,0 & 0,99 & 83,3 & 0,60 & 0,93 & 50,4 & 11 \\
\hline 8,0 & 0,99 & 87,0 & 0,53 & 0,95 & 51,6 & 11 \\
\hline
\end{tabular}

Este modelo supone que la superficie del adsorbente es heterogénea y que los sitios de adsorción tienen distintas afinidades; en primer lugar, se ocupan las posiciones de mayor afinidad y, posteriormente, se va ocupando el resto.

En la figura 2, se representa el ajuste de los datos experimentales a la isoterma de Freundlich y en la tabla 2, se muestran los valores obtenidos de los parámetros de este modelo. Se observa que los valores de para todos los $\mathrm{pH}$ son mayores que la unidad, lo que indica que el proceso de adsorción es favorable (Wang et al. 2006).

Isoterma de Langmuir: El modelo de Langmuir fue originalmente desarrollado para representar la adsorción de un sistema gas-sólido con carbón activado, pero ha sido ampliamente utilizado para estudiar la adsorción de un soluto en fase líquida. En este modelo, la atracción entre las moléculas y la superficie del adsorbente se basa, fundamentalmente, en fuerzas físicas y en su aplicación se asume, por un lado, que la adsorción ocurre en sitios activos idénticos de la superficie del adsorbente con energías uniformes de adsorción $y$, por otro, que una vez el soluto ocupa un lugar, no puede ocurrir otra adsorción en este mismo sitio. La isoterma de Langmuir, se puede representar de la siguiente manera (Langmuir, 1916):

$$
q=\left(\frac{q_{\max } b C_{e}}{1+b C_{e}}\right)
$$

donde $q_{\max }$ y $b$ son las constantes de Langmuir y representan la capacidad máxima de adsorción de la fase sólida $\left(\mathrm{mg} \mathrm{g}^{-1}\right)$ y la constante de energía relacionada con el calor de adsorción, respectivamente $\left(\mathrm{dm}^{3} \mathrm{mg}^{-1}\right)$. Esta ecuación, se puede escribir de forma lineal de la siguiente manera:

$\frac{C_{e}}{q}=\frac{1}{b q_{\operatorname{mac}}}+\frac{C_{e}}{q_{\max }}$
En la figura 3, se muestra el ajuste de los datos experimentales a la isoterma de Langmuir y en la tabla 2 aparecen los valores de los parámetros de este modelo obtenidos. Se observa que $q_{\max }$ está en el rango de 76,3 a $87,0 \mathrm{mg} \mathrm{g}^{-1} \mathrm{y}$ $b$ entre 0,64 y $0,53 \mathrm{dm}^{3} \mathrm{mg}^{-1}$. De acuerdo con los resultados obtenidos, el carbón activado se podría emplear como un adsorbente efectivo y considerarse como una alternativa para la remoción de colorantes (Satish et al. 2011).

Para determinar el modelo de isoterma que mejor se ajustará a los datos de equilibrio experimentales, se comparó los coeficientes de correlación de ambos modelos. Los valores de $R^{2}$ para las isotermas de Langmuir y Freundlich fueron 0,99 y 0,95 , respectivamente a $\mathrm{pH} 8$, similar comportamiento se muestra para los otros $\mathrm{pH}$ estudiados. Esto indica que la isoterma de Langmuir se ajusta mejor al proceso de adsorción que la isoterma de Freundlich. Resultados similares han sido reportados para la adsorción del colorante azul de metileno sobre carbón activado, preparado a partir de residuos de madera (Hameed et al. 2007), de salvado de trigo deshidratado (Özner \& Dursun, 2007), del tallo del algodón (Deng et al. 2011), de igual manera, para carbón activado comercial (Karaca et al. 2008; Orfão et al. 2006).

Se puede asumir, entonces, que en el CAG predominan los sitios activos que tienen una energía de adsorción uniforme, que estimulan la formación de monocapas de moléculas de AM, debido a las fuerzas electrostáticas (Din et al. 2009). Pero el ajuste satisfactorio al modelo de Freundlich sugiere que también existen sitios activos que presentan una distribución heterogénea de la energía de adsorción, que tienden a formar multicapas de moléculas de AM sobre el CAG, posiblemente, a la adsorción dimérica del AM sobre el carbón activado (Karaca et al. 2008).

Efecto del pH: El pH inicial de la disolución es una de las variables importantes en el proceso de adsorción porque afecta su extensión, debido a que influye en el grado de io- 


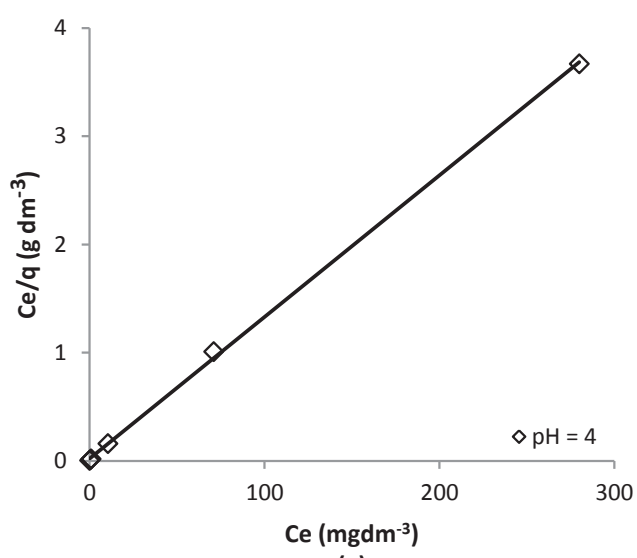

(a)

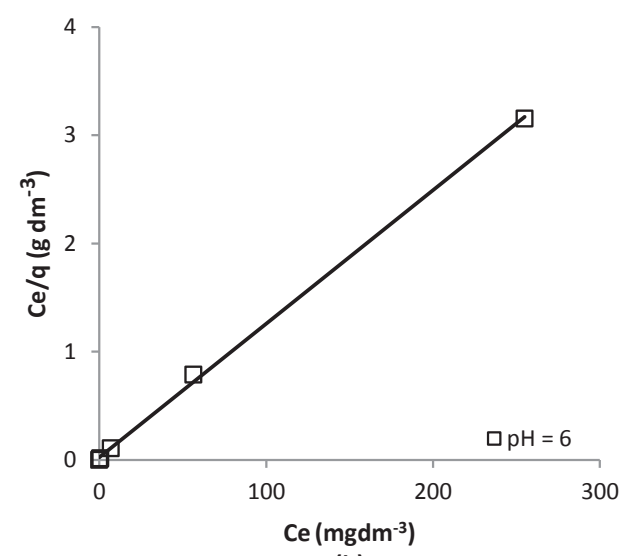

(b)

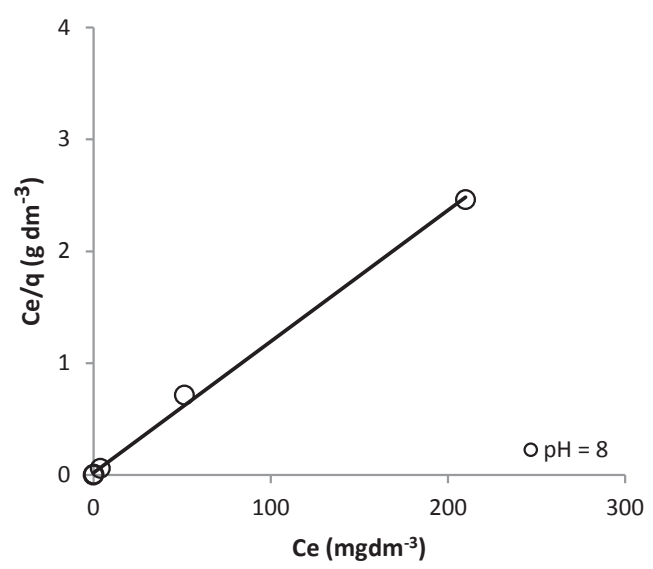

(c)

Figura 3. Comparación entre la ecuación linealizada del modelo de Langmuir y los datos experimentales a diferentes pH

nización de los compuestos ácidos y básicos (Karaca et al. 2008), pero además, modifica la carga de la superficie del carbón activado, aumentando o disminuyendo la capacidad de adsorción del mismo (Luo et al. 2006). Por lo tanto, en este estudio, se emplearon cinco diferentes $\mathrm{pH}(4,5,6,7 \mathrm{y}$ 8), para determinar su efecto en el equilibrio de adsorción.

La figura 4 muestra la relación entre la cantidad máxima de AM removido por unidad de masa de $\mathrm{CAG}$ y el $\mathrm{pH}$ inicial de la disolución. Como se observa, a pH 4 la capacidad de adsorción es de 76,3 $\mathrm{mg} \mathrm{g}^{-1}$, mientras que, a pH 8 es de $87,0 \mathrm{mg}$ $\mathrm{g}^{-1}$, el cual, muestra una relación directa entre el $\mathrm{pH}$ de la disolución y la capacidad de adsorción.

Este comportamiento, se puede explicar si se considera que en la región ácida la superficie del CAG se protona por el exceso de iones $\mathrm{H}^{+}$, que produce una carga neta positiva $y$, en consecuencia, los sitios activos del CAG generan una repulsión sobre los cationes del colorante AM, pero además, la presencia de iones $\mathrm{H}^{+}$en la disolución compiten con los cationes de AM por los sitios de unión del CAG y como resultado, se reduce el valor de la capacidad de adsorción. En la región básica, se registra la presencia de iones hidroxilos que promueven las atracciones electrostáticas con la carga positiva del colorante, mejorando, de esta manera, la capacidad de adsorción del adsorbente (Gobi et al. 2011).

Independiente del pH de la solución, la adsorción de AM sobre carbón activado, se favorece ampliamente, debido, en primer lugar, a las fuerzas de dispersión y a las interacciones entre los electrones $\pi$ de los sitios ácidos de Lewis en el plano basal del carbón activado y los electrones libres de las moléculas de AM presentes en los anillos aromáticos y enlaces múltiples y, en segundo lugar, a los enlaces de hidrógeno (Orfão et al. 2006).

Este trabajo de investigación sobre la adsorción del colorante catiónico AM sobre carbón activado granular en disolución acuosa, como una función de la concentración inicial y el $\mathrm{pH}$, permite concluir que cuando aumenta el $\mathrm{pH}$ y la 


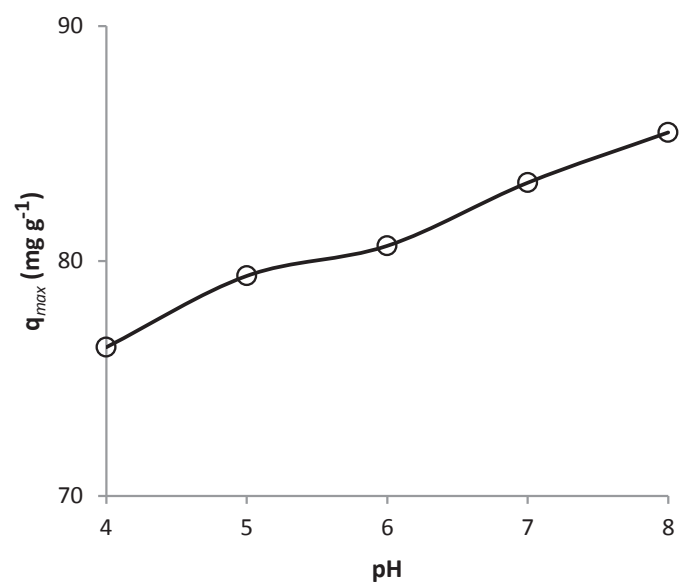

Figura 4. Efecto del pH sobre la capacidad máxima de adsorción del CAG.

concentración inicial de la disolución, mejora el proceso de adsorción. Los datos de equilibrio se ajustan significativamente al modelo de isoterma de Langmuir, que reporta una capacidad máxima de adsorción de $87,0 \mathrm{mg} \mathrm{g}^{-1}$ y junto con los valores de mayores que la unidad, para todos los $\mathrm{pH}$ estudiados, muestran que el carbón activado comercial empleado favorece la adsorción del colorante AM.

Agradecimientos: Los autores de este artículo agradecen la colaboración del Grupo de Investigación en Química Ambiental de la Universidad Autónoma del Caribe y al Grupo de Investigación en Biotecnología de Microalgas de la Universidad del Atlántico. Conflictos de intereses: El manuscrito fue preparado y revisado con la participación de todos los autores, quienes declaramos que no existe ningún conflicto de intereses que ponga en riesgo la validez de los resultados presentados.

\section{BIBLIOGRAFÍA}

1. AHMAD, M.A.; ALROZI, R. 2010. Optimization of preparation conditions for mangosteen peel-based activated carbons for the removal of Remazol Brilliant Blue $\mathrm{R}$ using response surface methodology. Chem. Eng. J. 165(3):883-890.

2. AHMAD, A.L.; LOH, M.M.; AZIZ, J.A. 2007. Preparation and characterization of activated carbon from oil palm wood and its evaluation on methylene blue adsorption. Dyes Pigm. 75(2):263-272.

3. AHMAD, A.A.; HAMEED, B.H. 2010. Fixed-bed adsorption of reactive azo dye onto granular activated carbon prepared from waste. J. Haz. Mat. 175(1-3):298-303.
4. BANSAL, R.C.; DONNET, J.B.; STOECKLI, F. 1988. Activated Carbon, Ed. Marcel Dekker (New York). 482p.

5. BASKARALINGAM, P.; PULIKESI, M.; RAMAMURTHI, V.; SIVANESAN, S. 2007. Modified hectorites and adsorption studies of a reactive dye-technical note. Appl. Clay Sci. 37(1-2):207-214.

6. BRLANUER, S.; EMMETT, P.H.; TELLER, E. 1938. Adsorption of gases in multimolecular layers. J. Am. Chem. Soc. 60(2):309-316.

7. BOEHM, H.P. 1994. Some aspects of the surface chemistry of carbon blacks and other carbons. Carbon. 32(5):759-769.

8. CASTELLAR, G.C.; GARCÍA, A.A. 2011. Remoción de $\mathrm{Pb}^{2+}$ en disolución acuosa sobre carbón activado en polvo: Estudio por lote. Prospectiva. 9(1):59-68.

9. CHATTERJEE, S.; CHATTERJEE, S.; CHATTERJEE, B.P.; DAS, A.R.; GUHA, A.K. 2005. Adsorption of a model anionic dye, eosin $\mathrm{Y}$, from aqueous solution by chitosan hydrobeads. J. Coll. Interf. Sci. 288(1):3035.

10. CHIOU, M.S.; LI, H.Y. 2003. Adsorption behavior of reactive dye in aqueous solution on chemical crosslinked chitosan beads. Chemosph. 50(8):1095-1105.

11. CHOY, K.K.H.; PORTER, J.F.; MCKAY, G. 2004. Intraparticle diffusion in single and multicomponent acid dye adsorption from wastewater onto carbon. Chem. Eng. J. 103(1-3):133-145. 
12. DAFALE, N.; WATE, S.; MESHRAM, S.; NANDY, T. 2008. Kinetic study approach of remazol black-B use for the development of two-stage anoxic-oxic reactor for decolorization biodegradation of azo dyes by activated bacterial consortium. J. Haz. Mat. 159(1-3):319328.

13. DENG, H; LU, J.; LI, G.; ZHANG, G.; WANG, X. 2011. Adsorption of methylene blue on adsorbent materials produced from cotton stalk. Chem. Eng. J. 172(1):326-334.

14. DÍAZ-VELÁSQUEZ, J. de J.; CARBALLO-SUÁREZ, L.M.; FIGUEIREDO, J.L. 2007.Thermal treatments of activated carbon catalysts under $\mathrm{N}_{2} \mathrm{O}$. Carbon. 45(1):212-214.

15. DIN, A.T.M.; HAMEED, B.H.; AHMAD, A.L. 2009. Batch adsorption of phenol onto physiochemical-activated coconut shell. J. Haz. Mat. 161(2-3):1522-1529.

16. FREUNDLICH, H.M.F. 1906. Over the adsorption in solution. J. Phys. Chem. 57:385-471.

17. GOBI, K.; MASHITAH, M.D.; VADIVELU, V.M. 2011. Adsorptive removal of Methylene Blue using novel adsorbent from palm oil mill effluent waste activated sludge: Equilibrium, thermodynamics and kinetic studies. Chem. Eng. J. 171(3):1246-1252.

18. HO, Y.S.; HUANG, C.T.; HUANG, H.W. 2002. Equilibrium sorption isotherm for metals ions on tree fern. Process Biochem. 37(12):1421-1430.

19. HAMEED, B.H.; AHMAD, A.L.; LATIFF, K.N.A. 2007. Adsorption of basic (methylene blue) onto activated carbon prepared from rattan sawdust. Dyes Pigments. 75(1):143-149.

20. HAMEED, B.H.; EL-KHAIARY, M.I. 2008. Equilibrium, kinetics and mechanism of malachite green adsorption activated carbon prepared from bamboo by $\mathrm{K}_{2} \mathrm{CO}_{3}$ activation and subsequent gasification with $\mathrm{CO}_{2}$. J. Haz. Mat. 157(2-3):344-351.

21. KARACA, S.; GÜRSES, A.; AÇIKYILDIZ, M.; EJDER, M. 2008. Adsorption of cationic dye from aqueous solutions by activated carbon. Micropor. Mesopor. Mat. 115(3):376-382.

22. LANGMUIR, I. 1916. The constitution and fundamental properties of solids and liquids. J. Am. Chem. Soc. 38:2221-2295.
23. LUO, S.L.; YUAN, L.; CHAI, L.Y.; MIN, X.B.; WANG, Y.Y.; WANG, P.; FANG, Y. 2006. Biosorption behaviors of $\mathrm{Cu}^{2+}, \mathrm{Zn}^{2+}, \mathrm{Cd}^{2+}$ and mixture by waste activated sludge. Transact. Nonferrous Met. Soc. China. 16(6):1431-1435.

24. MALIK, P.K. 2003. Use of activated carbons prepared from sawdust and rice-husk for adsorption of acid dyes: a case study of Acid Yellow 36. Dyes Pigm. 56(3):239-249.

25. ÓRFÃO, J.J.M.; SILVA, A.I.M.; PEREIRA, J.C.V.; BARATA, S.A.; FONSECA, I.M.; FARIA, P.C.C.; PEREIRA, M.F.R. 2006. Adsorption of a reactive dye on chemically modified activated carbons-influence of $\mathrm{pH}$. Colloid Interface Sci. 296(2):480-489.

26. ÖZNER, A.; DURSUN, G. 2007. Removal of methylene blue from aqueous solution by dehydrated wheat bran carbon. J. Haz. Mat. 146(1-2):262-269.

27. PEÑA, S.C.; TOBÓN, P.Y. 2006. Remoción del color de lodos provenientes de la industria textil por Aspergillus sp. Rev. Universidad Eafit. 4(142):88-94.

28. RAMAKRISHNA, K.; VIRARAGHAVAN, T. 1997. Use of slag for dye removal. Waste Manag. 17(8):483-488.

29. ROBINSON, I.M.; MCMULLAN, G.; MARCHANT, R.; NIGAM, P. 2001. Remediation of dyes in textile effluent: a critical review on current treatment technologies. Biores. Techn. 77(3):247-255.

30. SATISH, P.; SAMEER, R.; NASEEMA. P. 2011. Removal of methylene blue, a basic dye from aqueous solutions by adsorption using teak tree (Tectona grandis) bark powder. Int. J. Environ. Sci. 1(5):711-726.

31. SHI, Q.; ZHANG, J.; ZHANG, Ch., LI, C.; ZHANG, B.; HU, W.; XU, J.; ZHAO, R. 2010. Preparation of activated carbon from cattail and its application for dyes removal. J. Environ. Sci. 22(1):91-97.

32. SHI, B.Y.; LI, G.H.; WANG, D.S.; FENG, C.H.; TANG, H.X. 2007. Removal of direct dyes by coagulation: the performance of performance polymeric aluminum species. J. Haz. Mat. 143: 567-574.

33. SUHAS; CARROTT, P.J.M.; RIBEIRO CARROTT, M.M.L. 2007. Lignin-from natural adsorbent to activated carbon: A review. Biores. Techn. 98(12):2301-2312. 
34. TUNC, Ö.; TANACI, H.; AKZU, Z. 2009. Potential use of cotton plant waste for the removal Black $B$ reactive dye. J. Haz. Mat. 163(1):87-198.

35. WANG, S.; BOYJOO, Y.; CHOUEIB, A. 2005. A comparative study of dye removal using fly ash treated by different methods. Chemosph. 60(10):1401-1407.

36. WANG, Y.; MU, Y.; ZHAO, Q.B.; YU, H.Q. 2006. Isotherms, kinetics and thermodynamics of dye bio- sorption by anaerobic sludge. Separat. Purif. Techn. 50(1):1-7.

37. WACHOWSKI, L.; SOBCZAK, J.W.; HOFMAN, M. 2007. Speciation of functional groups formed on the surface of ammoxidised carbonaceous materials by XPS method. Appl. Surf. Sci. 253(9):4456-4461.

Recibido: Agosto 28 de 2012

Aceptado: Marzo 11 de 2013 